\title{
A New Generalization of Extended Beta and Hypergeometric Functions
}

\author{
Gauhar Rahman ${ }^{1}$, Kottakkaran Sooppy Nisar ${ }^{2}$ and Shahid Mubeen ${ }^{3}$ \\ ${ }^{1}$ Department of Mathematics, \\ International Islamic University, Islamabad, Pakistan \\ Email- gauhar55uom@gmail.com \\ ${ }^{2}$ Department of Mathematics, College of Arts and Science-Wadi Aldawaser, \\ Prince Sattam bin Abdulaziz University, Saudi Arabia \\ Email- n.sooppy@psau.edu.sa \\ ${ }^{3}$ Department of Mathematics, \\ University of Sargodha, Sargodha, Pakistan \\ Email-smjhanda@gmail.com
}

\begin{abstract}
A new generalization of extended beta function and its various properties, integral representations and distribution are given in this paper. In addition, we establish the generalization of extended hypergeometric and confluent hypergeometric functions using the newly extended beta function. Some properties of these extended and confluent hypergeometric functions such as integral representations, Mellin transformations, differentiation formulas, transformation and summation formulas are also investigated.
\end{abstract}

Keywords: Beta function; extended beta function; hypergeometric function; extended hypergeometric function; confluent hypergeometric function; extended confluent hypergeometric function; Mellin transform; beta distribution; transformation formula; summation formula.

AMS Subject Classification: 33B20, 33C20, 33C45, 33C60, 33B15, 33C05

\section{INTRODUCTION}

Recently many researchers introduced various extensions and generalizations of various special functions due to its applications in various fields. The latest development and properties of such extension is found in the recent work of various researchers (see e.g., $[1,2,3,4,7,9,12,11,13,14])$.

The extended beta function due to Chaudhry et al. [5] is defined by

$$
B\left(\varsigma_{1}, \varsigma_{2} ; p\right)=B_{p}\left(\varsigma_{1}, \varsigma_{2}\right)=\int_{0}^{1} t^{\varsigma_{1}-1}(1-t)^{\varsigma_{2}-1} e^{-\frac{p}{t(1-t)}} d t
$$


2

(where $\Re(p)>0, \Re\left(\varsigma_{1}\right)>0, \Re\left(\varsigma_{2}\right)>0$ ) respectively. When $p=0$, then $B\left(\varsigma_{1}, \varsigma_{2} ; 0\right)=$ $B\left(\varsigma_{1}, \varsigma_{2}\right)$, the well known beta function and is defined by

$$
B\left(\varsigma_{1}, \varsigma_{2}\right)=\int_{0}^{1} t^{\varsigma_{1}-1}(1-t)^{\varsigma_{2}-1} d t,\left(\Re\left(\varsigma_{1}\right)>0, \Re\left(\varsigma_{2}\right)>0\right),
$$

and

$$
B\left(\varsigma_{1}, \varsigma_{2}\right)=\frac{\Gamma\left(\varsigma_{1}\right) \Gamma\left(\varsigma_{2}\right)}{\Gamma\left(\varsigma_{1}+\varsigma_{2}\right)}, \Re\left(\varsigma_{1}\right)>0, \Re\left(\varsigma_{2}\right)>0 .
$$

In the same paper [5], another form of extended beta function is given by

$$
f(t)=\left\{\begin{array}{l}
\frac{1}{B_{p}(\varsigma, \beta)} t^{\varsigma-1}(1-t)^{\beta-1} \exp \left[-\frac{p}{t(1-t)}\right], 0<t<1 \\
0, \text { otherwise. }
\end{array}\right.
$$

The extended hypergeometric and confluent hypergeometric functions by using the definition of extended beta function $B_{p}(x, y)$ as follows (see [6] )

$$
F_{p}\left(\varsigma_{1}, \varsigma_{2} ; \varsigma_{3} ; z\right)=\sum_{n=0}^{\infty} \frac{B_{p}\left(\varsigma_{2}+n, \varsigma_{3}-\varsigma_{2}\right)}{B\left(\varsigma_{2}, \varsigma_{3}-\varsigma_{2}\right)}\left(\varsigma_{1}\right)_{n} \frac{z^{n}}{n !}
$$

where $p \geq 0$ and $\Re\left(\varsigma_{3}\right)>\Re\left(\varsigma_{2}\right)>0,|z|<1$ and

$$
\Phi_{p}\left(\varsigma_{2} ; \varsigma_{3} ; z\right)=\sum_{n=0}^{\infty} \frac{B_{p}\left(\varsigma_{2}+n, \varsigma_{3}-\varsigma_{2}\right)}{B\left(\varsigma_{2}, \varsigma_{3}-\varsigma_{2}\right)} \frac{z^{n}}{n !}
$$

where $p \geq 0$ and $\Re\left(\varsigma_{3}\right)>\Re\left(\varsigma_{2}\right)>0$.

And they defined the following integral representations of extended hypergeometric and confluent hypergeometric functions,

$$
\begin{aligned}
& F_{p}\left(\varsigma_{1}, \varsigma_{2} ; \varsigma_{3} ; z\right)=\frac{1}{B\left(\varsigma_{2}, \varsigma_{3}-\varsigma_{2}\right)} \\
& \times \int_{0}^{1} t^{\varsigma_{2}-1}(1-t)^{\varsigma_{3}-\varsigma_{2}-1}(1-z t)^{-\varsigma_{1}} \exp \left(\frac{-p}{t(1-t)}\right) d t, \\
&\left(p \geq 0, \Re\left(\varsigma_{3}\right)>\Re\left(\varsigma_{2}\right)>0,|\arg (1-z)|<\pi\right),
\end{aligned}
$$

and

$$
\begin{gathered}
\Phi_{p}\left(\varsigma_{2} ; \varsigma_{3} ; z\right)=\frac{1}{B\left(\varsigma_{2}, \varsigma_{3}-\varsigma_{2}\right)} \int_{0}^{1} t^{\varsigma_{2}-1}(1-t)^{\varsigma_{3}-\varsigma_{2}-1} \exp \left(z t-\frac{-p}{t(1-t)}\right) d t \\
\left(p \geq 0, \Re\left(\varsigma_{3}\right)>\Re\left(\varsigma_{2}\right)>0\right) .
\end{gathered}
$$


It is clear that when $p=0$, then the equations (1.4)-(1.7) reduce to the well known hypergeometric and confluent hypergeometric series and their integral representation respectively (see (1.8)-(1.11)) and the details are given as follows:

The Gauss hypergeometric and confluent hypergeometric functions which are defined (see [15]) as

$$
{ }_{2} F_{1}\left(\varsigma_{1}, \varsigma_{2} ; \varsigma_{3} ; z\right)=\sum_{n=0}^{\infty} \frac{\left(\varsigma_{1}\right)_{n}\left(\varsigma_{2}\right)_{n}}{\left(\varsigma_{3}\right)_{n}} \frac{z^{n}}{n !},(|z|<1),
$$

$\left(\varsigma_{1}, \varsigma_{2}, \varsigma_{3} \in \mathbb{C}\right.$ and $\left.\varsigma_{3} \neq 0,-1,-2,-3, \cdots\right)$, and

$$
{ }_{1} \Phi_{1}\left(\varsigma_{2} ; \varsigma_{3} ; z\right)=\sum_{n=0}^{\infty} \frac{\left(\varsigma_{2}\right)_{n}}{\left(\varsigma_{3}\right)_{n}} \frac{z^{n}}{n !},(|z|<1),
$$

$\left(\varsigma_{2}, \varsigma_{3} \in \mathbb{C}\right.$ and $\left.\varsigma_{3} \neq 0,-1,-2,-3, \cdots\right)$, respectively.

The integral representation of hypergeometric and confluent hypergeometric functions are respectively defined by

$$
\begin{gathered}
{ }_{2} F_{1}\left(\varsigma_{1}, \varsigma_{2} ; \varsigma_{3} ; z\right)=\frac{\Gamma\left(\varsigma_{3}\right)}{\Gamma\left(\varsigma_{2}\right) \Gamma\left(\varsigma_{3}-\varsigma_{2}\right)} \int_{0}^{1} t^{\varsigma_{2}-1}(1-t)^{\varsigma_{3}-\varsigma_{2}-1}(1-z t)^{-\varsigma_{1}} d t, \\
\left(\Re\left(\varsigma_{3}\right)>\Re\left(\varsigma_{2}\right)>0,|\arg (1-z)|<\pi\right), \text { and } \\
{ }_{1} \Phi_{1}\left(\varsigma_{2} ; \varsigma_{3} ; z\right)=\frac{\Gamma\left(\varsigma_{3}\right)}{\Gamma\left(\varsigma_{2}\right) \Gamma\left(\varsigma_{3}-\varsigma_{2}\right)} \int_{0}^{1} t^{\varsigma_{2}-1}(1-t)^{\varsigma_{3}-\varsigma_{2}-1} e^{z t} d t, \\
\left(\Re\left(\varsigma_{3}\right)>\Re\left(\varsigma_{2}\right)>0\right) .
\end{gathered}
$$

Very recently Shadab et al. [17] introduced a new and modified extension of beta function defined by

$$
B_{p}^{\lambda}\left(\varsigma_{1}, \varsigma_{2}\right)=\int_{0}^{1} t^{\varsigma_{1}-1}(1-t)^{\varsigma_{2}-1} E_{\lambda}\left(-\frac{p}{t(1-t)}\right) d t
$$

where $\Re\left(\varsigma_{1}\right)>0, \Re\left(\varsigma_{2}\right)>0$ and $E_{\lambda}($.$) is Mittag-Leffler function defined by$

$$
E_{\lambda}(z)=\sum_{n=0}^{\infty} \frac{z^{n}}{\Gamma(\lambda n+1)}
$$

Obviously, when $\lambda=1$ then $B_{p}^{1}\left(\varsigma_{1}, \varsigma_{2}\right)=B_{p}\left(\varsigma_{1}, \varsigma_{2}\right)$ is the extended beta function (see[5]). Similarly, when when $\lambda=1$ and $p=0$, then $B_{0}^{1}\left(\varsigma_{1}, \varsigma_{2}\right)=B_{0}\left(\varsigma_{1}, \varsigma_{2}\right)$ is the classical beta function. 
They [17] also defined extended hypergeometric function and its integral representation

$$
\begin{aligned}
F_{p}^{\lambda}\left(\varsigma_{1}, \varsigma_{2} ; \varsigma_{3} ; z\right)={ }_{2} F_{1}\left(\varsigma_{1}, \varsigma_{2} ; \varsigma_{3} ; z ; p, \lambda\right) & =\sum_{n=0}^{\infty}\left(\varsigma_{1}\right)_{n} \frac{B_{p}^{\lambda}\left(\varsigma_{2}+n, \varsigma_{3}-\varsigma_{2}\right)}{B\left(\varsigma_{2}, \varsigma_{3}-\varsigma_{2}\right)} \frac{z^{n}}{n !} \\
= & \sum_{n=0}^{\infty}\left(\varsigma_{1}\right)_{n} \frac{B\left(\varsigma_{2}+n, \varsigma_{3}-\varsigma_{2} ; p, \lambda\right)}{B\left(\varsigma_{2}, \varsigma_{3}-\varsigma_{2}\right)} \frac{z^{n}}{n !}
\end{aligned}
$$

where $p \geq 0, \lambda>0, \varsigma_{1}, \varsigma_{2}, \varsigma_{3} \in \mathbb{C}$ and $|z|<1$.

$$
\begin{aligned}
F_{p}^{\lambda}\left(\sigma_{1}, \sigma_{2} ; \sigma_{3} ; z\right) & =\frac{1}{B\left(\varsigma_{2} ; \varsigma_{3}-\varsigma_{2}\right)} \\
& \times \int_{0}^{1} t^{\varsigma_{2}-1}(1-t)^{\varsigma_{3}-\varsigma_{2}-1}(1-t z)^{-\varsigma_{1}} E_{\lambda}\left(-\frac{p}{t(1-t)}\right) d t,
\end{aligned}
$$

where $\Re(p)>0, \Re(\lambda)>0, \Re\left(\varsigma_{3}\right)>\Re\left(\varsigma_{2}\right)>0$.

The extended confluent hypergeometric function and its integral representation involving (1.12) is defined by

$$
\begin{aligned}
\Phi_{p}^{\lambda}\left(\varsigma_{2} ; \varsigma_{3} ; z\right)=\Phi\left(\varsigma_{1}, \varsigma_{2} ; \varsigma_{3} ; z ; p, \lambda\right) & =\sum_{n=0}^{\infty} \frac{B_{p}^{\lambda}\left(\varsigma_{2}+n, \varsigma_{3}-\varsigma_{2}\right)}{B\left(\varsigma_{2}, \varsigma_{3}-\varsigma_{2}\right)} \frac{z^{n}}{n !} \\
& =\sum_{n=0}^{\infty} \frac{B\left(\varsigma_{2}+n, \varsigma_{3}-\varsigma_{2} ; p, \lambda\right)}{B\left(\varsigma_{2}, \varsigma_{3}-\varsigma_{2}\right)} \frac{z^{n}}{n !}
\end{aligned}
$$

where $p \geq 0, \lambda>0, \sigma_{2}, \sigma_{3} \in \mathbb{C}$, and

$$
\Phi_{p}^{\lambda}\left(\varsigma_{2} ; \varsigma_{3} ; z\right)=\frac{1}{B\left(\varsigma_{2}, \varsigma_{3}-\varsigma_{2}\right)} \int_{0}^{1} t^{\varsigma_{2}-1}(1-t)^{\varsigma_{3}-\varsigma_{2}-1} \exp (t z) E_{\lambda}\left(-\frac{p}{t(1-t)}\right) d t
$$

where $\Re(p)>0, \Re(\lambda)>0, \Re\left(\varsigma_{3}\right)>\Re\left(\varsigma_{2}\right)>0$.

Obviously when $\lambda=1$, then (1.14)-(1.17) will reduce to the extended hypergeometric function (1.4)-(1.7) and similarly when $\lambda=1$ and $p=0$ then (1.14)-(1.17) will reduce to (1.8)-(1.11).

Also, in [17] authors defined the extended beta function as:

$$
f(t)=\left\{\begin{array}{l}
\frac{1}{B_{p}^{\lambda}\left(\varsigma_{1}, \varsigma_{2}\right)} t^{\varsigma_{1}-1}(1-t)^{\varsigma_{2}-1} E_{\lambda}\left(-\frac{p}{t(1-t)}\right), 0<t<1 \\
0, \text { otherwise }
\end{array}\right.
$$

\section{A NEW GENERALIZATION OF EXTENDED BETA FUNCTION AND ITS PROPERTIES}

In this section, we establish a new generalization of extended beta function and its properties such as Mellin transforms and integral representations. 
Definition 2.1. The generalization of extended beta function is defined as:

$$
B_{p}^{\lambda ; m}(\varsigma, \beta)=B(x, y ; p ; \lambda ; m)=\int_{0}^{1} z^{\varsigma-1}(1-z)^{\beta-1} E_{\lambda}\left(-\frac{p}{z^{m}(1-z)^{m}}\right) d z,
$$

where $\Re(\varsigma)>0, \Re(\beta)>0, \Re(p) \geq 0$ and $\lambda, m>0$ and $E_{\lambda}^{p}($.$) is Mittag-Leffler function.$

Remark 2.1. Note that:

(i) If $\lambda=1$, then (2.1) reduces the generalized extended beta function given in [16].

(ii) If $m=1$, then (2.1) reduces to the well known extended beta function (1.12).

(iii) If $m=\lambda=1$, then (2.1) reduces to the well known extended beta function (1.1).

(iv)If $p=0$ and $\lambda=m=1$, then (2.1) reduces to the classical beta function (1.2).

Theorem 1. The extension of extended beta function have the following Millin transform relation:

$$
M\left\{B_{p}^{\lambda ; m}(\varsigma, \beta) ; p \rightarrow s\right\}=\frac{\pi}{\sin (\pi s) \Gamma(1-s \lambda)} B(\varsigma+m s, \beta+m s),
$$

where $\Re(s)>0, \Re(\varsigma+m s)>0$ and $\Re(\beta+m s)>0$.

Proof. Applying the Mellin transform on (2.1), we have

$$
M\left\{B_{p}^{\lambda ; m}(\varsigma, \beta) ; p \rightarrow s\right\}=\int_{0}^{\infty} p^{s-1}\left(\int_{0}^{1} z^{\varsigma-1}(1-z)^{\beta-1} E_{\lambda}\left(-\frac{p}{z^{m}(1-z)^{m}}\right)\right) d z d p
$$

Interchanging the order integrations, we have

$$
M\left\{B_{p}^{\lambda ; m}(\varsigma, \beta) ; p \rightarrow s\right\}=\int_{0}^{1} z^{\varsigma-1}(1-z)^{\beta-1}\left\{\int_{0}^{\infty} p^{s-1} E_{\lambda}\left(-\frac{p}{z^{m}(1-z)^{m}}\right) d p\right\} d z
$$

Substituting $v=\frac{p}{z^{m}(1-z)^{m}}$ in (2.3), we have

$$
M\left\{B_{p}^{\lambda ; m}(\varsigma, \beta) ; p \rightarrow s\right\}=\int_{0}^{1} z^{\varsigma+m s-1}(1-z)^{\beta+m s-1}\left\{\int_{0}^{\infty} v^{s-1} E_{\lambda}(-v) d w .\right.
$$

By using the following formula,

$$
\int_{0}^{\infty} v^{s-1} E_{\lambda, \gamma}^{\delta}(-w v) d v=\frac{\Gamma(s) \Gamma(\delta-s)}{\Gamma(\delta) w^{s} \Gamma(\gamma-s \lambda)},
$$

for $\gamma=\delta=1$ and $w=1(2.5)$ becomes

$$
\int_{0}^{\infty} v^{s-1} E_{\lambda, \gamma}^{\delta}(-w v) d v=\frac{\Gamma(s) \Gamma(1-s)}{\Gamma(1-s \lambda)}
$$

By applying the (2.6) to (2.4), we have

$$
M\left\{B_{p}^{\lambda ; m}(\varsigma, \beta) ; p \rightarrow s\right\}=\frac{\Gamma(s) \Gamma(1-s)}{\Gamma(1-s \lambda)} B(\varsigma+m s, \beta+m s) .
$$


6

Now, using the Euler's reflection formula on Gamma function,

$$
\Gamma(s) \Gamma(1-s)=\frac{\pi}{\sin (\pi s)},
$$

we get the desired result.

Corollary 2.1. The following integral representation holds true

$$
\int_{0}^{\infty} B_{p}(\varsigma, \beta) d p=B(\varsigma+1, \beta+1)
$$

Proof. By taking $s=1$ and $\lambda=m=1$ in Theorem 1, we get the required result.

Theorem 2. The following integral representations holds true

$$
\begin{gathered}
B_{p}^{\lambda ; m}(\varsigma, \beta)=2 \int_{0}^{\frac{\pi}{2}} \cos ^{2 \varsigma-1} \theta \sin ^{2 \beta-1} \theta E_{\lambda}\left(-\frac{p}{\cos ^{2 m} \theta \sin ^{2 m} \theta}\right) d \theta \\
B_{p}^{\lambda ; m}(\varsigma, \beta)=\int_{0}^{\infty} \frac{u^{\varsigma-1}}{(1+u)^{\varsigma+\beta}} E_{\lambda}\left(-\frac{p(1+u)^{2 m}}{u^{m}}\right) d u \\
B_{p}^{\lambda}(\varsigma, \beta)=2^{1-\varsigma-\beta} \int_{-1}^{1}(1+u)^{\varsigma-1}(1-u)^{\beta-1} E_{\lambda}\left(-\frac{4^{m} p}{\left(1-u^{2}\right)^{m}}\right) d u
\end{gathered}
$$

and

$$
\begin{aligned}
B_{p}^{\lambda}(x, y)= & (c-a)^{1-\varsigma-\beta} \int_{a}^{c}(u-a)^{\varsigma-1}(c-u)^{\beta-1} E_{\lambda}\left(-\frac{p(c-a)^{2 m}}{(u-a)^{m}(c-u)^{m}}\right) d u, \\
& (p, \geq 0, \lambda, m>0, \Re(p)>0, \Re(\lambda)>0, \Re(\varsigma)>0, \Re(\beta)>0) .
\end{aligned}
$$

Proof. Equations (2.8)-(2.11) can be easily obtained by taking the transformation $t=$ $\cos ^{2} \theta, t=\frac{u}{1+u}, t=\frac{1+u}{2}$ and $t=\frac{u-a}{c-a}$ in (2.1), respectively.

\section{PROPERTIES OF GENERALIZED EXTENDED BETA FUNCTION}

In this section, we investigate various properties of (2.1).

Theorem 3. The extension of beta function satisfies the following integral representation

$$
B_{p}^{\lambda ; m}(\varsigma+1, \beta)+B_{p}^{\lambda ; m}(\varsigma, \beta+1)=B_{p}^{\lambda ; m}(\varsigma, \beta) .
$$

Proof. Consider the left hand side of (3.1), we have

$$
\begin{aligned}
B_{p}^{\lambda ; m}(\varsigma+1, \beta) & +B_{p}^{\lambda ; m}(\varsigma, \beta+1) \\
& =\int_{0}^{1}\left[t^{\varsigma}(1-t)^{\beta-1}+t^{\varsigma-1}(1-t)^{\beta}\right] E_{\lambda}\left(-\frac{p}{t^{m}(1-t)^{m}}\right) d t
\end{aligned}
$$

which proves the desired result. 
Corollary 3.1. The following result holds true

$$
B_{p}^{\lambda}(\varsigma+1, \beta)+B_{p}^{\lambda}(\varsigma, \beta+1)=B_{p}^{\lambda}(\varsigma, \beta) .
$$

Proof. Setting $m=1$ in Theorem 3, we get the desired result.

Corollary 3.2. The following result holds true

$$
B_{p}(\varsigma+1, \beta)+B_{p}(\varsigma, \beta+1)=B_{p}(\varsigma, \beta) .
$$

Proof. Setting $m=\lambda=1$ in Theorem 3, we get the desired result.

Corollary 3.3. The following integral representation holds true

$$
B(\varsigma+1, \beta)+B(\varsigma, \beta+1)=B(\varsigma, \beta) .
$$

Proof. Setting $m=\lambda=1$ and $p=0$ in Theorem 3, we get the required result.

Theorem 4. The extension of beta function satisfies the following summation formulas

$$
B_{p}^{\lambda ; m}(\varsigma, 1-\beta)=\sum_{n=0}^{\infty} \frac{(\beta)_{n}}{n !} B_{p}^{\lambda ; m}(\varsigma+n, 1),(\Re(p)>0, m, \lambda>0) .
$$

Proof. Consider the generalized binomial theorem

$$
(1-t)^{-\beta}=\sum_{n=0}^{\infty}(\beta)_{n} \frac{t^{n}}{n !}(|t|<1) .
$$

Applying (3.6) to the definition (2.1) of extended beta function

$$
B_{p}^{\lambda ; m}(\varsigma, 1-\beta)=\int_{0}^{1} \sum_{n=0}^{\infty} \frac{(\beta)_{n} t^{\varsigma+n-1}}{n !} E_{\lambda}\left(-\frac{p}{t^{m}(1-t)^{m}}\right) d t
$$

Now, interchanging the order of summation and integration in above equation and using (2.1) proves the desired result.

Theorem 5. The extension of beta function satisfies the following infinite summation formulas

$$
B_{p}^{\lambda ; m}(\varsigma, \beta)=\sum_{n=0}^{\infty} B_{p}^{\lambda ; m}(\varsigma+n, \beta+1),(\Re(p)>0, \lambda>0) .
$$

Proof. Replacing the following series representation in (2.1)

$$
(1-t)^{\beta-1}=(1-t)^{\beta} \sum_{n=0}^{\infty} t^{n}
$$


8

we obtain

$$
B_{p}^{\lambda ; m}(\varsigma, \beta)=\int_{0}^{1}(1-t)^{\beta} \sum_{n=0}^{\infty} t^{\varsigma+n-1} E_{\lambda}\left(-\frac{p}{t^{m}(1-t)^{m}}\right) d t .
$$

By interchanging the order of integration and summation in above equation and using (2.1), we get the desired result.

Theorem 6. The following relation holds true

$$
B_{p}^{\lambda ; m}(a,-a-n)=\sum_{k=0}^{n}\left(\begin{array}{l}
n \\
k
\end{array}\right) B_{p}^{\lambda ; m}(a+k,-a-k),\left(n \in \mathbb{N}_{0}\right) .
$$

Proof. The fundamental relation gives

$$
B_{p}^{\lambda ; m}\left(\varsigma_{1}+1, \varsigma_{2}\right)+B_{p}^{\lambda ; m}\left(\varsigma_{1}, \varsigma_{2}+1\right)=B_{p}^{\lambda ; m}\left(\varsigma_{1}, \varsigma_{2}\right)
$$

Taking $\varsigma_{1}=a$ and $\varsigma_{2}=-a-n$ in above relation, we have

$$
B_{p}^{\lambda ; m}(a,-a-n)=B_{p}^{\lambda ; m}(a,-a-n+1)+B_{p}^{\lambda ; m}(a+1,-a-n) .
$$

Starting with $n=1,2,3 \cdots$, we have

$$
\begin{aligned}
B_{p}^{\lambda ; m}(a,-a-1) & =B_{p}^{\lambda ; m}(a,-a)+B_{p}^{\lambda ; m}(a+1,-a-1), \\
B_{p}^{\lambda ; m}(a,-a-2) & =B_{p}^{\lambda ; m}(a,-a)+2 B_{p}^{\lambda ; m}(a+1,-a-1)+B_{p}^{\lambda ; m}(a+2,-a-2), \\
B_{p}^{\lambda ; m}(a,-a-3) & =B_{p}^{\lambda ; m}(a,-a)+3 B_{p}^{\lambda ; m}(a+1,-a-1)+3 B_{p}^{\lambda ; m}(a+2,-a-2) \\
& +B_{p}^{\lambda ; m}(a+3,-a-3),
\end{aligned}
$$

and so on. The above series behaves like as finite binomials series does. Thus, we can finally obtain the desired relation (3.8).

Note that, we can also prove the desired inequality by applying induction on $n$.

Theorem 7. For $B_{p}^{\lambda ; m}(\varsigma, \beta)$, we have the following Mellin transformation formula:

$$
\begin{gathered}
B_{p}^{\lambda ; m}(\varsigma, \beta)=\frac{\pi}{2 \pi \iota} \int_{\gamma-\iota \infty}^{\gamma+\iota \infty} \frac{\Gamma(\varsigma+m s) \Gamma(\beta+m s)}{\sin (\pi s) \Gamma(1-s \lambda) \Gamma(\varsigma+\beta+2 m s)} p^{-s} d s \\
(\Re(p)>0, \Re(\lambda)>0, \gamma>0), m>0 .
\end{gathered}
$$

Proof. Applying the inverse Mellin transform on both sides of (2.2), we get the desired result. 


\section{The Generalized EXTENDED Beta Distribution}

In this section, we give a new generalization of extended beta distribution. Also, we define its mean, variance and moment generating function of the newly defined beta distribution:

$$
f(t)=\left\{\begin{array}{l}
\frac{1}{B_{p}^{\lambda ; m}(\varsigma, \beta)} t^{\varsigma-1}(1-t)^{\beta-1} E_{\lambda}\left(-\frac{p}{t^{m}(1-t)^{m}}\right), 0<t<1 \\
0, \text { otherwise }
\end{array}\right.
$$

If $\nu$ ia any real number, then the mean of extended beta distribution (4.1) is defined as;

$$
\begin{gathered}
E\left(X^{\nu}\right)=\frac{B_{p}^{\lambda ; m}(\varsigma+\nu, \beta)}{B_{p}^{\lambda ; m}(\varsigma, \beta)} \\
(p>0, m, \lambda>0,-\infty<\varsigma, \beta<\infty) .
\end{gathered}
$$

When $v=1$, then we get the mean of the distribution

$$
\mu=E(X)=\frac{B_{p}^{\lambda ; m}(\varsigma+1, \beta)}{B_{p}^{\lambda ; m}(\varsigma, \beta)} .
$$

The various of the distribution can defined by

$$
\begin{aligned}
\sigma^{2} & =E\left(X^{2}\right)-\{E(X)\}^{2} \\
& =\frac{B_{p}^{\lambda ; m}(\varsigma, \beta) B_{p}^{\lambda ; m}(\varsigma+2, \beta)-\left\{B_{p}^{\lambda ; m}(\varsigma+1, \beta)\right\}^{2}}{\left\{B_{p}^{\lambda ; m}(\varsigma, \beta)\right\}^{2}} .
\end{aligned}
$$

The moment generating function of the distribution is defined by

$$
M(t)=\sum_{n=0}^{\infty} \frac{t^{n}}{n !} E\left(X^{n}\right)=\frac{1}{B_{p}^{\lambda ; m}(\varsigma, \beta)} \sum_{n=0}^{\infty} B_{p}^{\lambda ; m}(\varsigma+n, \beta) \frac{t^{n}}{n !} .
$$

The cumulative distribution of (4.1) can be defined as

$$
F(x)=\frac{B_{x, p}^{\lambda ; m}(\varsigma, \beta)}{B_{p}^{\lambda ; m}(\varsigma, \beta)}
$$

where

$$
\begin{aligned}
B_{x, p}^{\lambda ; m}(\varsigma, \beta) & =\int_{0}^{x} t^{\varsigma-1}(1-t)^{\beta-1} E_{\lambda}\left(-\frac{p}{t^{m}(1-t)^{m}}\right) d t \\
& (p>0, m, \lambda>0,-\infty<\varsigma, \beta<\infty)
\end{aligned}
$$

is an extension of incomplete beta function. 
Remark 4.1. Obviously, if $m=1$ then the beta distribution defined in (4.1) reduces to the beta distribution defined by Shadab et al. [17]. Similarly $m=\lambda=1$, then the beta distribution defined in (4.1) reduces to the extended beta distribution defined by Chaudhry et al. [5].

\section{Generalization of Extended hypergeometric FunCtions}

In this section, we introduce generalization of a ne extension of extended hypergeometric and confluent hypergeometric functions by using the generalized extended beta function (2.1).

Definition 5.1. The generalization of extended hypergeometric function is defined as;

$$
F_{p}^{\lambda ; m}\left(\varsigma_{1}, \varsigma_{2} ; \varsigma_{3} ; z\right)=\sum_{n=0}^{\infty}\left(\varsigma_{1}\right)_{n} \frac{B_{p}^{\lambda ; m}\left(\varsigma_{2}+n ; \varsigma_{3}-\varsigma_{2}\right)}{B\left(\varsigma_{2} ; \varsigma_{3}-\varsigma_{2}\right)} \frac{z^{n}}{n !},
$$

where $p \geq 0, m, \lambda>0, \Re\left(\varsigma_{3}\right)>\Re\left(\varsigma_{2}\right)>0,|z|<1$ and $E_{\lambda}($.$) is Mittag-Leffler function.$

Definition 5.2. The generalization of extended confluent hypergeometric function is defined as:

$$
\Phi_{p}^{\lambda ; m}\left(\varsigma_{2} ; \varsigma_{3} ; z\right)=\sum_{n=0}^{\infty} \frac{B_{p}^{\lambda ; m}\left(\varsigma_{2}+n ; \varsigma_{3}-\varsigma_{2}\right)}{B\left(\varsigma_{2} ; \varsigma_{3}-\varsigma_{2}\right)} \frac{z^{n}}{n !},
$$

where $p \geq 0, m, \lambda>0, \Re\left(\varsigma_{3}\right)>\Re\left(\varsigma_{2}\right)>0$.

Remark 5.1. It is clear that

(i) If $\lambda=1$, then (5.1) and (5.2) reduces to the extended hypergeometric and confluent hypergeometric functions given in [16].

(ii) if we letting $m=1$, then (5.1) and (5.2) reduce to the extended hypergeometric and confluent hypergeometric functions (1.14) and (1.16) respectively.

(iii) if we letting $m=\lambda=1$, then (5.1) and (5.2) reduce to the extended hypergeometric and confluent hypergeometric functions (1.4) and (1.5) respectively.

(iv) if we letting $\lambda=1$ and $p=0$, then (5.1) and (5.2) reduce to the classical hypergeometric and confluent hypergeometric functions (1.8) and (1.9) respectively.

\section{INTEGRAL REPRESENTATIONS OF GENERALIZED EXTENDED HYPERGEOMETRIC FUNCTIONS}

In this section, we define the integral representations of (5.1) and (5.2).

Theorem 8. The extended hypergeometric has the following integral representation;

$$
F_{p}^{\lambda ; m}\left(\varsigma_{1}, \varsigma_{2} ; \varsigma_{3} ; z\right)=\frac{1}{B\left(\varsigma_{2}, \varsigma_{3}-\varsigma_{2}\right)}
$$




$$
\times \int_{0}^{1} t^{\varsigma_{2}-1}(1-t)^{\varsigma_{3}-\varsigma_{2}-1}(1-t z)^{-\varsigma_{1}} E_{\lambda}\left(-\frac{p}{t^{m}(1-t)^{m}}\right) d t
$$

where $m, p, \lambda>0, p=0, \Re\left(\varsigma_{3}\right)>\Re\left(\varsigma_{2}\right)>0$ and $|\arg (1-z)|<\pi$.

Proof. By using (2.1) in (5.1), we have

$$
\begin{aligned}
F_{p}^{\lambda ; m}\left(\varsigma_{1}, \varsigma_{2} ; \varsigma_{3} ; z\right) & =\frac{1}{B\left(\varsigma_{2}, \varsigma_{3}-\varsigma_{2}\right)} \\
& \times \int_{0}^{1} t^{\varsigma_{2}-1}(1-t)^{\varsigma_{3}-\varsigma_{2}-1} E_{\lambda}\left(-\frac{p}{t^{m}(1-t)^{m}}\right) \sum_{n=0}^{\infty}\left(\varsigma_{1}\right)_{n} \frac{(z t)^{n}}{n !} d t
\end{aligned}
$$

Thus by using $\sum_{n=0}^{\infty}\left(\varsigma_{1}\right)_{n} \frac{(z t)^{n}}{n !}=(1-t z)^{-\varsigma_{1}}$ in $(6.2)$, we get the desired result.

Theorem 9. The following integral representations holds true:

$$
\begin{aligned}
F_{p}^{\lambda ; m}\left(\varsigma_{1}, \varsigma_{2} ; \varsigma_{3} ; z\right) & =\frac{1}{B\left(\varsigma_{2}, \varsigma_{3}-\varsigma_{2}\right)} \\
& \times \int_{0}^{1} u^{\varsigma_{2}-1}(1+u)^{\varsigma_{1}-\varsigma_{3}}(1-u(1+z))^{-\varsigma_{1}} E_{\lambda}\left(-\frac{p(1+u)^{2 m}}{u^{m}}\right) d t \\
F_{p}^{\lambda ; m}\left(\varsigma_{1}, \varsigma_{2} ; \varsigma_{3} ; z\right) & =\frac{2}{B\left(\varsigma_{2}, \varsigma_{3}-\varsigma_{2}\right)} \\
& \times \int_{0}^{\frac{\pi}{2}} \frac{(\sin \theta)^{2 \varsigma_{2}-1}(\cos \theta)^{2 \varsigma_{3}-2 \varsigma_{2}-1}}{\left(1-z \sin ^{2} \theta\right)^{\varsigma_{1}}} E_{\lambda}\left(-p \sec ^{2 m} \theta \csc ^{2 m} \theta\right) d \theta
\end{aligned}
$$

and

$$
\begin{aligned}
F_{p}^{\lambda ; m}\left(\varsigma_{1}, \varsigma_{2} ; \varsigma_{3} ; z\right) & =\frac{2}{B\left(\varsigma_{2}, \varsigma_{3}-\varsigma_{2}\right)} \\
& \times \int_{0}^{\infty} \frac{(\sinh \theta)^{2 \varsigma_{2}-1}(\cosh \theta)^{2 \varsigma_{1}-2 \varsigma_{3}-1}}{\left(\cosh ^{2} \theta-z \sinh ^{2} \theta\right)^{\varsigma_{1}}} E_{\lambda}\left(-p \cosh ^{2 m} \theta \operatorname{coth}^{2 m} \theta\right) d \theta
\end{aligned}
$$

Proof. By substituting $t=\frac{u}{1+u}, t=\sin ^{2} \theta$ and $t=\tanh ^{2} \theta$ in (5.1) respectively, we get the desired representations (6.3)-(6.5)

Next, we prove integral representations of extended confluent hypergeometric function.

\section{Theorem 10.}

$$
\begin{aligned}
\Phi_{p}^{\lambda ; m}\left(\varsigma_{2} ; \varsigma_{3} ; z\right) & =\frac{1}{B\left(\varsigma_{2} ; \varsigma_{3}-\varsigma_{2}\right)} \\
& \times \int_{0}^{1} t^{\varsigma_{2}-1}(1-t)^{\varsigma_{3}-\varsigma_{2}-1} \exp (z t) E_{\lambda}\left(-\frac{p}{t^{m}(1-t)^{m}}\right) d t
\end{aligned}
$$


12

and

$$
\begin{aligned}
\Phi_{p}^{\lambda ; m}\left(\varsigma_{2} ; \varsigma_{3} ; z\right) & =\frac{\exp (z)}{B\left(\varsigma_{2} ; \varsigma_{3}-\varsigma_{2}\right)} \\
& \times \int_{0}^{1}(1-t)^{\varsigma_{2}-1} t^{\varsigma_{3}-\varsigma_{2}-1} \exp (-z t) E_{\lambda}\left(-\frac{p}{t^{m}(1-t)^{m}}\right) d t
\end{aligned}
$$

Proof. By using definition of extended beta function (2.1) in (5.2), we have

$$
\begin{aligned}
\Phi_{p}^{\lambda ; m}\left(\varsigma_{2} ; \varsigma_{3} ; z\right) & =\frac{1}{B\left(\varsigma_{2} ; \varsigma_{3}-\varsigma_{2}\right)} \\
& \times \int_{0}^{1} t^{\varsigma_{2}-1}(1-t)^{\varsigma_{3}-\varsigma_{2}-1} E_{\lambda}\left(-\frac{p}{t^{m}(1-t)^{m}}\right)\left(\sum_{n=0}^{\infty} \frac{(z t)^{n}}{n !}\right) d t
\end{aligned}
$$

Using $\sum_{n=0}^{\infty} \frac{(z t)^{n}}{n !}=\exp (z t)$ in (6.8), we get the proof of $(6.6)$.

To prove (6.7), substituting $t=1-t$ in (6.6).

7. DifFERENTIATION FORMulas FOR THE EXTENDED HyPERGEOMETRIC FUNCTIONS

In this section, we derive the differentiations formulas for the generalized extended hypergeometric and confluent hypergeometric functions.

\section{Theorem 11.}

$$
\frac{d^{n}}{d z^{n}}\left\{F_{p}^{\lambda ; m}\left(\varsigma_{1}, \varsigma_{2} ; \varsigma_{3} ; z\right)\right\}=\frac{\left(\varsigma_{1}\right)_{n}\left(\varsigma_{2}\right)_{n}}{\left(\varsigma_{3}\right)_{n}} F_{p}^{\lambda ; m}\left(\varsigma_{1}+n, \varsigma_{2}+n ; \varsigma_{3}+n ; z\right)
$$

Proof. Differentiating (5.1) with respect to $z$, we have

$$
\begin{aligned}
\frac{d}{d z}\left\{F_{p}^{\lambda ; m}\left(\varsigma_{1}, \varsigma_{2} ; \varsigma_{3} ; z\right)\right\} & =\frac{d}{d z} \sum_{n=0}^{\infty}\left(\varsigma_{1}\right)_{n} \frac{B_{p}^{\lambda ; m}\left(\varsigma_{2}+n ; \varsigma_{3}-\varsigma_{2}\right)}{B\left(\varsigma_{2}, \varsigma_{3}-\varsigma_{2}\right)} \frac{z^{n}}{n !} \\
& =\sum_{n=1}^{\infty}\left(\varsigma_{1}\right)_{n} \frac{B_{p}^{\lambda ; m}\left(\varsigma_{2}+n ; \varsigma_{3}-\varsigma_{2}\right)}{B\left(\varsigma_{2}, \varsigma_{3}-\varsigma_{2}\right)} \frac{z^{n-1}}{(n-1) !} .
\end{aligned}
$$

Changing $n$ to $n+1$ in (7.2), we have

$$
\frac{d}{d z}\left\{F_{p}^{\lambda ; m}\left(\varsigma_{1}, \varsigma_{2} ; \varsigma_{3} ; z\right)\right\}==\sum_{n=0}^{\infty}\left(\varsigma_{1}\right)_{n+1} \frac{B_{p}^{\lambda ; m}\left(\varsigma_{2}+n+1 ; \varsigma_{3}-\varsigma_{2}\right)}{B\left(\varsigma_{2}, \varsigma_{3}-\varsigma_{2}\right)} \frac{z^{n}}{n !} .
$$

Since

$$
B(b, c-b)=\frac{c}{b} B(b+1, c-b)
$$

Applying (7.4) to (7.3), we get

$$
\frac{d}{d z}\left\{F_{p}^{\lambda ; m}\left(\varsigma_{1}, \varsigma_{2} ; \varsigma_{3} ; z\right)\right\}=\frac{\varsigma_{1} \varsigma_{2}}{\varsigma_{3}} \sum_{n=0}^{\infty}\left(\varsigma_{1}+1\right)_{n} \frac{B_{p}^{\lambda ; m}\left(\varsigma_{2}+n+1 ; \varsigma_{3}-\varsigma_{2}\right)}{B\left(\varsigma_{2}+1, \varsigma_{3}-\varsigma_{2}\right)} \frac{z^{n}}{n !}
$$




$$
=\frac{\varsigma_{1} \varsigma_{2}}{\varsigma_{3}} F_{p}^{\lambda ; m}\left(\varsigma_{1}+1, \varsigma_{2}+1 ; \varsigma_{3}+1 ; z\right) .
$$

Again differentiating (7.5) with respect to $z$, we obtain

$$
\frac{d^{2}}{d z^{2}}\left\{F_{p}^{\lambda ; m}\left(\varsigma_{1}, \varsigma_{2} ; \varsigma_{3} ; z\right)\right\}=\frac{\varsigma_{1}\left(\varsigma_{1}+1\right) \varsigma_{2}\left(\varsigma_{2}+1\right)}{\varsigma_{3}\left(\varsigma_{3}+1\right)} F_{p}^{\lambda ; m}\left(\varsigma_{1}+2, \varsigma_{2}+2 ; \varsigma_{3}+2 ; z\right) .
$$

Continuing up to $n$ times, we get the required result.

\section{Theorem 12.}

$$
\frac{d^{n}}{d z^{n}}\left\{\Phi_{p}^{\lambda ; m}\left(\varsigma_{2} ; \varsigma_{3} ; z\right)\right\}=\frac{\left(\varsigma_{2}\right)_{n}}{\left(\varsigma_{3}\right)_{n}} \Phi_{p}^{\lambda ; m}\left(\varsigma_{2}+n ; \varsigma_{3}+n ; z\right) .
$$

Proof. Applying the similar procedure used in Theorem 11, we get the desired result.

\section{Mellin transformation of EXtended hypergeOMETRiC FUnCTIONS}

In this section, we derive the Mellin transformation of extended hypergeometric and confluent hypergeometric functions (5.1) and (5.2).

Theorem 13. The extended hypergeometric function has the following Mellin transform;

$$
\begin{aligned}
M\left\{F_{p}^{\lambda ; m}\left(\varsigma_{1}, \varsigma_{2} ; \varsigma_{3} ; z\right) ; p \rightarrow s\right\} & =\frac{\pi B\left(\varsigma_{2}+m s, \varsigma_{3}+m s-\varsigma_{2}\right)}{\sin (\pi s) \Gamma(1-s \lambda) B\left(\varsigma_{2}, \varsigma_{3}-\varsigma_{2}\right)} \\
& \times F\left(\varsigma_{1}, \varsigma_{2}+m s ; \varsigma_{3}+2 m s ; z\right),
\end{aligned}
$$

where $\Re\left(\varsigma_{2}+s\right)>0$, and $\Re\left(\varsigma_{3}+s\right)>0$.

Proof. Applying Mellin transform on both sides of (6.1), we have

$$
\begin{aligned}
& M\left\{F_{p}^{\lambda ; m}\left(\varsigma_{1}, \varsigma_{2} ; \varsigma_{3} ; z\right) ; p \rightarrow s\right\} \\
= & \frac{1}{B\left(\varsigma_{2}, \varsigma_{3}-\varsigma_{2}\right)} \int_{0}^{\infty} p^{s-1} \int_{0}^{1} t^{\varsigma_{2}-1}(1-t)^{\varsigma_{3}-\varsigma_{2}-1}(1-t z)^{-\varsigma_{1}} E_{\lambda}\left(-\frac{p}{t^{m}(1-t)^{m}}\right) d t d p
\end{aligned}
$$

Interchanging the order of integrations in above equation, we have

$$
\begin{aligned}
M\left\{F_{p}^{\lambda ; m}\left(\varsigma_{1}, \varsigma_{2} ; \varsigma_{3} ; z\right) ; p \rightarrow s\right\} & =\frac{1}{B\left(\varsigma_{2}, \varsigma_{3}-\varsigma_{2}\right)} \int_{0}^{1} t^{\varsigma_{2}-1}(1-t)^{\varsigma_{3}-\varsigma_{2}-1}(1-t z)^{-\varsigma_{1}} \\
& \times\left\{\int_{0}^{\infty} p^{s-1} E_{\lambda}\left(-\frac{p}{t^{m}(1-t)^{m}}\right) d p\right\} d t
\end{aligned}
$$

Letting $v=\frac{p}{t^{m}(1-t)^{m}},(8.2)$ reduces to

$$
\begin{aligned}
M\left\{F_{p}^{\lambda ; m}\left(\varsigma_{1}, \varsigma_{2} ; \varsigma_{3} ; z\right) ; p \rightarrow s\right\}= & \frac{1}{B\left(\varsigma_{2}, \varsigma_{3}-\varsigma_{2}\right)} \int_{0}^{1} t^{\varsigma_{2}+m s-1}(1-t)^{\varsigma_{3}+m s-\varsigma_{2}-1}(1-t z)^{-\varsigma_{1}} \\
& \times\left\{\int_{0}^{\infty} v^{s-1} E_{\lambda}(-v) d v\right\} d t
\end{aligned}
$$


using (2.6) in (8.3), we have

$$
\begin{aligned}
& M\left\{F_{p}^{\lambda ; m}\left(\varsigma_{1}, \varsigma_{2} ; \varsigma_{3} ; z\right) ; p \rightarrow s\right\} \\
= & \frac{\Gamma(s) \Gamma(1-s)}{\Gamma(1-s \lambda)} \frac{B\left(\varsigma_{2}+m s, \varsigma_{3}+m s-\varsigma_{2}\right)}{B\left(\varsigma_{2}, \varsigma_{3}-\varsigma_{2}\right)} F\left(\varsigma_{1}, \varsigma_{2}+m s ; \varsigma_{3}+2 m s ; z\right) .
\end{aligned}
$$

Now, using the Euler's reflection formula on Gamma function (2.7), we get the desired result.

Theorem 14. The following result holds true;

$$
\begin{aligned}
F_{p}^{\lambda ; m}\left(\varsigma_{1}, \varsigma_{2} ; \varsigma_{3} ; z\right) & =\frac{1}{2 \iota B\left(\varsigma_{2}, \varsigma_{3}-\varsigma_{2}\right)} \int_{\gamma-\iota \infty}^{\gamma+\iota \infty} \frac{\Gamma\left(\varsigma_{2}+m s\right) \Gamma\left(\varsigma_{3}+m s-\varsigma_{2}\right)}{\sin (\pi s) \Gamma\left(\varsigma_{3}+2 m s\right)} \\
& \times F\left(\varsigma_{1}, \varsigma_{2}+m s ; \varsigma_{3}+2 m s ; z\right) p^{-s} d s,(\gamma>0) .
\end{aligned}
$$

Proof. Taking the inverse Mellin transform of both sides on (8.1), we get the required result.

In similar way, we can prove the following theorems for extended confluent hypergeometric functions.

Theorem 15. The extended confluent hypergeometric function has the following Mellin transform;

$$
\begin{aligned}
M\left\{\Phi_{p}^{\lambda ; m}\left(\varsigma_{2} ; \varsigma_{3} ; z\right) ; p \rightarrow s\right\} & =\frac{\pi B\left(\varsigma_{2}+m s, \varsigma_{3}+m s-\varsigma_{2}\right)}{\sin (\pi s) \Gamma(1-s \lambda) B\left(\varsigma_{2}, \varsigma_{3}-\varsigma_{2}\right)} \\
& \times \Phi\left(\varsigma_{2}+m s ; \varsigma_{3}+2 m s ; z\right),
\end{aligned}
$$

where $\Re\left(\varsigma_{2}+s\right)>0$, and $\Re\left(\varsigma_{3}+s\right)>0$.

Theorem 16. The following result holds true;

$$
\begin{aligned}
\Phi_{p}^{\lambda ; m}\left(\varsigma_{2} ; \varsigma_{3} ; z\right) & =\frac{1}{2 \iota B\left(\varsigma_{2}, \varsigma_{3}-\varsigma_{2}\right)} \int_{\gamma-\iota \infty}^{\gamma+\iota \infty} \frac{\Gamma\left(\varsigma_{2}+m s\right) \Gamma\left(\varsigma_{3}+m s-\varsigma_{2}\right)}{\sin (\pi s) \Gamma(1-s \lambda) \Gamma\left(\varsigma_{3}+2 m s\right)} \\
& \times \Phi\left(\varsigma_{2}+m s ; \varsigma_{3}+2 m s ; z\right) p^{-s} d s,(\gamma>0) .
\end{aligned}
$$

\section{TRANSFORMATION AND SUMMATION FORMULAS}

In this section, we obtain transformation and summation formulas for the generalized extended hypergeometric and confluent hypergeometric functions as follows:

Theorem 17. The following transformation for extended hypergeometric function holds true for $p, \lambda>0$ :

$$
F_{p}^{\lambda ; m}\left(\varsigma_{1}, \varsigma_{2} ; \varsigma_{3} ; z\right)=(1-z)^{-\varsigma_{1}} F_{p}^{\lambda ; m}\left(\varsigma_{1}, \varsigma_{2} ; \varsigma_{3} ;-\frac{z}{1-z}\right),
$$


where $|\arg (1-z)|<\pi$.

Proof. Replacing $t$ by $(1-t)$ in (6.1), we get the desired result.

Theorem 18. The following transformation for extended confluent hypergeometric function holds true for $p, \lambda>0$ :

$$
\Phi_{p}^{\lambda ; m}\left(\varsigma_{1}, \varsigma_{2} ; \varsigma_{3} ; z\right)=\exp (z) \Phi_{p}^{\lambda ; m}\left(\varsigma_{3}-\varsigma_{2} ; \varsigma_{3} ;-z\right),
$$

where $|\arg (1-z)|<\pi$.

Proof. From (6.6) and (6.7), we established the required result.

Theorem 19. The following summation formula holds true

$$
F_{p}^{\lambda ; m}\left(\varsigma_{1}, \varsigma_{2} ; \varsigma_{3} ; z\right)=\frac{B_{p}^{\lambda ; m}\left(\varsigma_{2}, \varsigma_{3}-\varsigma_{1}-\varsigma_{2}\right)}{B\left(\varsigma_{2}, \varsigma_{3}-\varsigma_{2}\right)}
$$

where $p \geq 0, \lambda>0$ and $\Re\left(\varsigma_{3}-\varsigma_{1}-\varsigma_{2}\right)>0$.

Proof. Taking $z=1$ in (6.1), we have

$$
F_{p}^{\lambda ; m}\left(\varsigma_{1}, \varsigma_{2} ; \varsigma_{3} ; 1\right)=\frac{1}{B\left(\varsigma_{2}, \varsigma_{3}-\varsigma_{2}\right)} \int_{0}^{1} t^{\varsigma_{2}-1}(1-t)^{\varsigma_{3}-\varsigma_{1}-\varsigma_{2}-1} E_{\lambda}\left(-\frac{p}{t^{m}(1-t)^{m}}\right) d t
$$

By applying definition (2.1) to the above equation, we get the desired result.

\section{CONCluding REMARKS}

In this paper, we established the generalization of extended beta function and hypergeometric functions. In conclusion, by letting $\lambda=1$, the results obtained in this paper will reduces to the results of [16] and by letting $m=1$ throughout the paper, all the results will be reduced to the work of Shadab et al. [17]. Similarly, if we set $m=\lambda=1$ then the results obtained in this paper will give the results of Chaudhry et al. (see $[5,6]$ ). In a similar way, if we letting $m=\lambda=1$ and $p=0$ then all the results will be reduced to the results involving the classical beta function, beta distribution, Gauss hypergeometric and confluent hypergeometric functions etc (see [8], [15]).

\section{Conflict of Interests}

The author(s) declare(s) that there is no conflict of interests regarding the publication of this article. 


\section{REFERENCES}

[1] P. Agarwal, J. Choi, S. Jain, Extended hypergeometric functions of two and three varaibles, Commun. Korean Math. Soc. 30 (2015), No. 4, pp. 403-414.

[2] M. Arshad, S. Mubeen, K. S. Nisar, G. Rahman, Extended Wright-Bessel function and its properties, Commun. Korean Math. Soc. 33 (2018), No. 1, 143155.

[3] M. Arshad, J. Choi S. Mubeen, K. S. Nisar, G. Rahman, A new extension of the Mittag-Leffler function, Commun. Korean Math. Soc. 2018, (Accepted).

[4] M. A. Chaudhry, S. M. Zubair, Generalized incomplete gamma functions with applications, J. Comput. Appl. Math. 55, 99124, (1994).

[5] M. A. Chaudhry, A. Qadir, M. Rafique, S. M. Zubair,Extension of Eulers beta function, J. Comput. Appl. Math. 78 (1997) 19-32.

[6] M. A. Chaudhry, A. Qadir, H. M. Srivastava and R. B. Paris, Extended Hypergeometric and Confluent Hypergeometric functions, Appl. Math. Comput., 159 (2004) 589-602

[7] J. Choi, A. K. Rathie, R. K. Parmar, Extension of extended beta, hypergeometric and confluent hypergeometric functions, Honam Mathematical J. 36 (2014), No. 2, pp. 357-385.

[8] A. Erdélyi,W. Magnus, F. Oberhettinger, and F.G. Tricomi, Higher Transcendental Functions, Vol.1, McGraw-Hill, NewYork, Toronto, London, 1953.

[9] S. Mubeen, G. Rahman, K. S. Nisar, J. Choi. M. Arshad, An extended beta function and its properties, Far East Journal of Mathematical Sciences, 102(2017), 1545-1557.

[10] D. J. Maširević, R. K. Parmar, T. K. Pogány, $(p, q)$-Extended Bessel and Modified Bessel functions of the first kind, Results Math. 2017.

[11] E. Özergin, M. A. Özarslan, Abdullah Altn, Extension of gamma, beta and hypergeometric functions, J. Comput. Appl. Math., 235 (2011), 4601-4610.

[12] M. A. Özarslan, B. Ylmaz, The extended Mittag-Leffler function and its properties, Journal of Inequalities and Applications, 2014:85, (2014).

[13] R. K. Parmar, Extended $\tau$-hypergeometric functions and associated properties, C. R.Acad.Sci.Paris,Ser.I353(2015)421-426.

[14] R. K. Parmar, A new generalization of gamma, beta, hypergeometric and confluent hypergeometric functions, LE MATEMATICHE Vol. LXVIII (2013) Fasc. II, pp. 33-52

[15] E. D. Rainville, Special functions, The Macmillan Company, New York, 1960.

[16] D.M. Lee, A.K. Rathie, R.K. Parmar and Y.S. Kim, Generalization of extended beta function, hypergeometric and confluent hypergeometric functions, Honam Mathematical J. 33 (2011), No. 2, pp. 187-206

[17] M. Shadab, S. Jabee and J. Choi, An extension of beta function and its application, Far East Journal of Mathematical Sciences, 103(2018), No. 1, pp 235-251. 